\title{
Coriocarcinoma de placenta metastásico en embarazo de 34 semanas. Revisión y manejo
}

\author{
Metastatic placenta choriocarcinoma in a 34 week pregnancy. Revision and management
}

Blanca Moreno-Gomez*, Jesús Castillo-Aznar, Patricia Tejedor-Vargas, Juan A. Delgado-Domingo, Andrés Millaruelo-Rami, Marimar Pérez-Morente y Ana Ramón- Navarro

Servicio de Anestesiología, Reanimación y Terapéutica del Dolor, Hospital Universitario Miguel Servet, Zaragoza, España

\begin{abstract}
Resumen
El coriocarcinoma es un tumor de la placenta agresivo y poco frecuente que forma parte de las denominadas enfermedades trofoblásticas gestacionales. Presentamos el caso de una mujer de 22 años nulípara que, en el transcurso del embarazo, en la semana 34, presenta metrorragia con hemoptisis de forma espontánea. Se realiza una tomografía computarizada en la que se aprecian nódulos compatibles con metástasis, catalogándose como posible coriocarcinoma metastásico. Se programa para cesárea preferente y se inicia tratamiento quimioterápico. Este tumor es muy sensible a la quimioterapia y presenta un alto índice de supervivencia, aunque en los casos metastásicos puede ser mortal.
\end{abstract}

Palabras clave: Coriocarcinoma. Coriocarcinoma metastásico. Embarazo.

\begin{abstract}
Choriocarcinoma is a rare, aggressive placental tumor that is part of so-called gestational trophoblastic diseases. We present the case of a 22-year-old nulliparous woman who spontaneously presents metrorrhagia with hemoptysis in the course of pregnancy at the $34^{\text {th }}$ week. A tomography scan is performed to confirm nodules compatible with metastasis, cataloguing it as a possible metastatic choriocarcinoma. A cesarean section is preferably scheduled and treatment with chemotherapy is initiated. Choriocarcinoma is a tumor very sensitive to chemotherapy and has a high survival rate, although in metastatic cases the disease is usually fatal.
\end{abstract}

Key words: Choriocarcinoma. Metastatic choriocarcinoma. Pregnancy.

\section{Introducción}

El coriocarcinoma es un tumor agresivo de la placenta que forma parte de las enfermedades trofoblásticas gestacionales. Hasta en un $50 \%$ de los casos procede de la malignización de una mola hidatiforme, aunque existe otro porcentaje que deriva de un embarazo ectópico, un aborto o incluso un embarazo a término. Entre los factores de riesgo están los antecedentes de abortos, embarazo múltiple, nuliparidad o edad mayor de 35 años. La patogenia viene determinada por la invasión de las células del trofoblasto a la decidua con capacidad metastásica preferentemente

\footnotetext{
Correspondencia:

*Blanca Moreno-Gómez

Vía Hispanidad 25, escalera 1, 2.ำ Fecha de recepción: 30-06-2019

C.P. 50012, Zaragoza, España $\quad$ Fecha de aceptación: 12-01-2021

E-mail: blanmorenogomez@gmail.com DOI: 10.24875/CIRU.19001402

Cir Cir. 2021;89(4):534-537

Contents available at PubMed

www.cirugiaycirujanos.com

0009-7411/@ 2021 Academia Mexicana de Cirugía. Publicado por Permanyer. Este es un artículo open access bajo la licencia CC BY-NC-ND (http://creativecommons.org/licenses/by-nc-nd/4.0/).
} 
en los pulmones, aunque hay casos descritos con metástasis hepáticas y cerebrales. El cuadro clínico más frecuente incluye sangrado vaginal, hemoptisis, dolor en el pecho, náuseas y vómitos'. Destaca también una elevación de la fracción beta de la hormona gonadotrofina coriónica humana, que es un marcador tumoral base del diagnóstico y de la respuesta al tratamiento. La alta producción de gonadotrofina coriónica puede ser la causa de manifestaciones en otros órganos, como quistes tecaluteínicos en los ovarios, hiperplasia endometrial, hiperplasia de lobulillos mamarios e hipertiroidismo secundario al aumento de la producción de gonadotrofina ${ }^{2}$. El tratamiento principal es médico y se basa en la administración de metotrexato, ácido folínico, actinomicina $D$, etopósido o vincristina. Desde el punto de vista quirúrgico, la histerectomía se realiza cuando hay resistencia a la quimioterapia. La tasa de supervivencia es del $85-94 \%$ si no existe enfermedad metastásica. Con metástasis, la supervivencia disminuye según el órgano afectado: un $70 \%$ en caso de afectación cerebral, un $27 \%$ si es el hígado y un $10 \%$ cuando están afectados ambos.

\section{Caso clínico}

Mujer de 22 años, embarazada de 34 semanas, sin alergias ni otros antecedentes de interés. Embarazo bien controlado y con parámetros y controles todos ellos dentro de la normalidad. Acude a urgencias por presentar metrorragia y hemoptisis de forma espontánea, con ligera disnea. En el servicio de urgencias se realiza una exploración física en la que destacan taquicardia, hipoventilación pulmonar basal derecha y restos de sangre en la vagina, sin otros hallazgos de interés. Se solicitan analítica de sangre con bioquímica, fracción beta de la gonadotrofina coriónica humana ( $\beta$-hCG) y hormonas tiroideas, hemograma, pruebas de coagulación y radiografía de tórax.

La analítica de sangre muestra una hemoglobina de $9.5 \mathrm{~g} / \mathrm{dl}, \beta$-hCG de $267,789 \mathrm{mUl} / \mathrm{ml}$ y hormonas tiroideas elevadas con disminución de la hormona estimulante del tiroides; el resto de los parámetros dentro de la normalidad. La radiografía de tórax se informa con abundantes infiltrados compatibles con metástasis más derrame pulmonar de predominio derecho (Fig. 1). Ante los hallazgos se solicita tomografía computarizada, en la que se objetivan abundantes nódulos en ambos pulmones con componente necrótico (Fig. 2) y placenta posterior con captación heterogénea y crecimientos nodulares/mamelonados (Fig. 3),

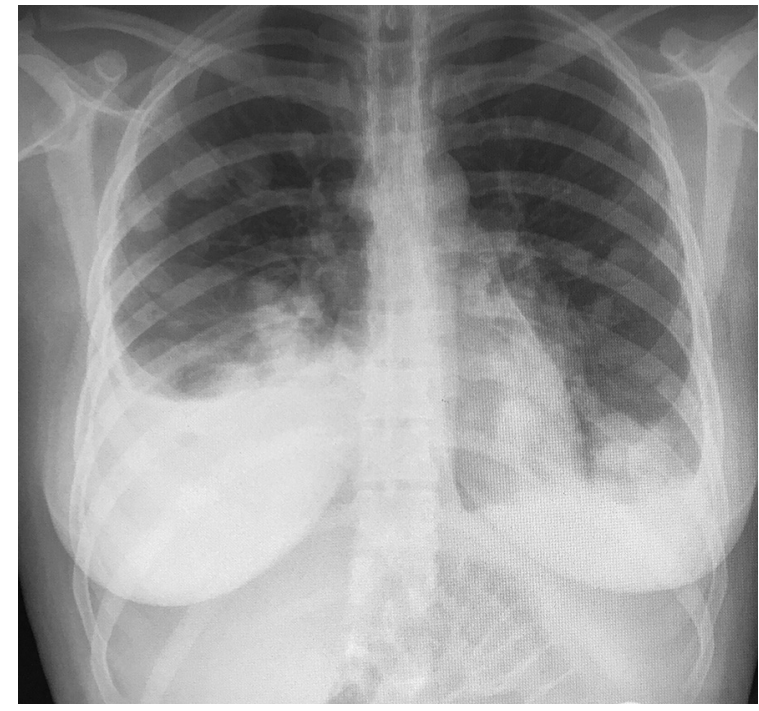

Figura 1. Radiografía de tórax que muestra infiltrados pulmonares bilaterales compatibles con metástasis con derrame pulmonar de predominio derecho.

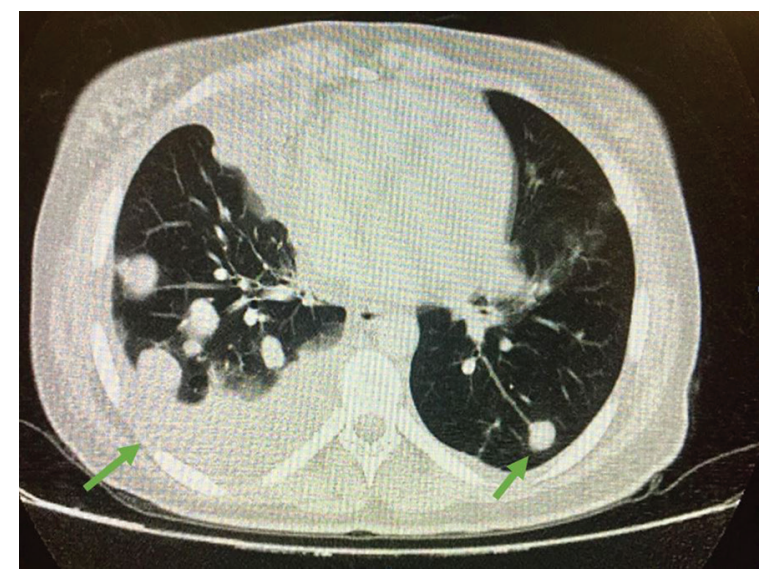

Figura 2. Tomografía computarizada pulmonar que muestra múltiples lesiones sospechosas de metástasis, la mayoría rodeadas de halo en "vidrio deslustrado" y de captación heterogénea, probablemente necróticas, con atelectasia en el lóbulo medio derecho.

todo ello compatible con metástasis de coriocarcinoma a confirmar por anatomía patológica.

Dado el embarazo, el deseo de un futuro nuevo embarazo y la gravedad del cuadro, el equipo interdisciplinario, formado por dos anestesiólogos y dos ginecólogos, decide realizar una cesárea programada de forma preferente para iniciar tratamiento con quimioterapia lo antes posible.

Antes de la cirugía se realiza tratamiento con propiltiouracilo y propanolol para estabilizar la tiroides y evitar una crisis tirotóxica, y se administra betametasona 


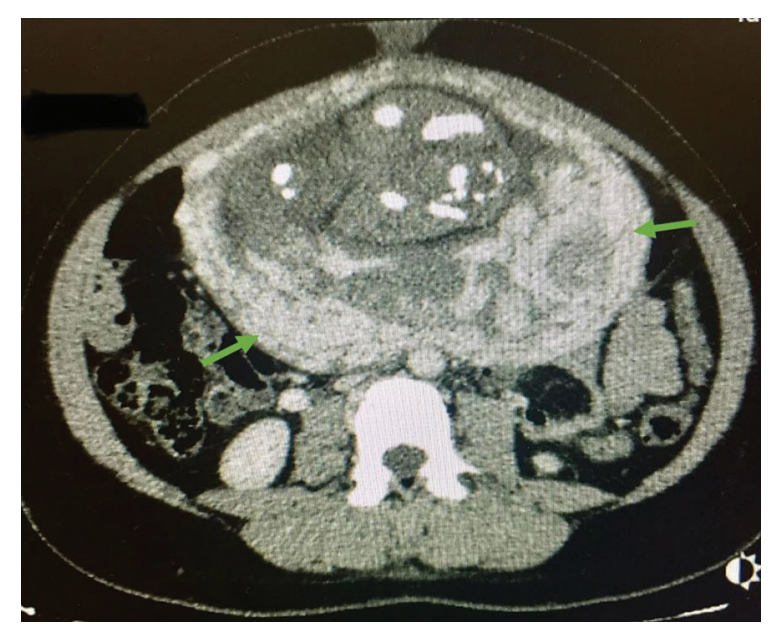

Figura 3. Placenta en posición posterior, de hasta $4 \mathrm{~cm}$ de espesor. Presenta una densidad heterogénea, con formaciones nodulares $y$ mamelonadas de predominio superior.

intramuscular para completar la maduración pulmonar. Por la posibilidad de sangrado, se realizan pruebas cruzadas y se reservan tres unidades de concentrados de hematíes.

El día de la cirugía se realiza nueva analítica de sangre, que muestra una hemoglobina de $8 \mathrm{mg} / \mathrm{dl}$ y un hematocrito del $25 \%$, con el resto de los parámetros dentro de la normalidad. Se decide realizar una transfusión de una unidad de concentrado de glóbulos rojos y se canalizan dos vías periféricas gruesas (16 G). Se monitoriza a la paciente con pulsioxímetro, presión arterial y electrocardiograma, y se realiza anestesia subdural con bupivacaína hiperbara al $0.5 \%(11 \mathrm{mg})$ y morfina $(0,1 \mathrm{mg})$. Tras la anestesia, la paciente presenta hipotensión que se resuelve con una infusión de fenilefrina a razón de $50 \mu \mathrm{g} / \mathrm{min}$. Comienza la cirugía con incisión de Pfannenstiel y se extrae al feto. La cirugía transcurre sin incidencias y al finalizar se realiza bloqueo plano transverso del abdomen bilateral con levobupivacaína al $0.25 \%$ (30 ml) y se traslada a la paciente a la unidad de reanimación, con evolución favorable y alta a las 48 horas a la unidad de oncología, donde una vez confirmado el diagnóstico por anatomía patológica se inicia tratamiento con el esquema EMACO (etopósido $100 \mathrm{mg} / \mathrm{m}^{2}$ los días 1-2, actinomicina $D \quad 0,5 \mathrm{mg}$ los días $1-2$, metotrexato $300 \mathrm{mg} / \mathrm{m}^{2}$ el día 1 , vincristina $1 \mathrm{mg} / \mathrm{m}^{2}$ el día 8 y ciclofosfamida $600 \mathrm{mg} / \mathrm{m}^{2}$ el día 8, en ciclos repetidos cada 14 días).

Los valores de $\beta$-hCG descendieron desde el inicio del tratamiento: $191,702.31 \mathrm{mUl} / \mathrm{l}$ a los 3 días, $36,353.73 \mathrm{mUl} / \mathrm{l}$ a los 7 días y $80.38 \mathrm{mUl} / \mathrm{l}$ al mes, hasta negativizarse tras la administración de seis
Tabla 1. Clasificación FIGO (Federación Internacional de Ginecología y Obstetricia)

\begin{tabular}{ll}
\hline Estadio & Criterio \\
\hline Estadio I & Enfermedad intraútero \\
Estadio II & $\begin{array}{l}\text { Tumor trofoblástico gestacional que se extiende fuera } \\
\text { del útero, pero que se limita a los órganos genitales } \\
\text { (anejos, vagina, ligamentos anchos) }\end{array}$ \\
Estadio III & $\begin{array}{l}\text { Enfermedad trofoblástica gestacional que se extiende } \\
\text { hasta los pulmones, con o sin afectación de vías } \\
\text { genitales conocida }\end{array}$ \\
Estadio IV & \begin{tabular}{l} 
Todos los otros sitios de metástasis \\
\hline
\end{tabular}
\end{tabular}

Tabla 2. Puntuación pronóstica

\begin{tabular}{|c|c|c|c|c|}
\hline & 0 & 1 & 2 & 4 \\
\hline Edad & $\begin{array}{l}\text { Hasta } 39 \\
\text { años }\end{array}$ & $\begin{array}{l}\text { Más de } 40 \\
\text { años }\end{array}$ & - & - \\
\hline $\begin{array}{l}\text { Antecedentes de } \\
\text { embarazo }\end{array}$ & $\begin{array}{l}\text { Mola } \\
\text { hidatiforme }\end{array}$ & $\begin{array}{l}\text { Aborto/ } \\
\text { desconocido }\end{array}$ & Término & - \\
\hline $\begin{array}{l}\text { Tamaño tumoral } \\
(\mathrm{cm})\end{array}$ & - & $3-5 \mathrm{~cm}$ & $>5 \mathrm{~cm}$ & - \\
\hline $\begin{array}{l}\text { Fracción beta } \\
\text { gonadotrofina } \\
\text { coriónica humana }\end{array}$ & $10^{3}$ & $10^{3}-10^{4}$ & $10^{4}-10^{5}$ & $>10^{5}$ \\
\hline $\begin{array}{l}\text { Fallo de } \\
\text { quimioterapia } \\
\text { anterior }\end{array}$ & - & - & Un fármaco & $\begin{array}{l}\text { Dos o } \\
\text { más } \\
\text { fármacos }\end{array}$ \\
\hline $\begin{array}{l}\text { Lugar de } \\
\text { metástasis }\end{array}$ & $\begin{array}{l}\text { Pulmón } \\
\text { Vagina }\end{array}$ & $\begin{array}{l}\text { Riñón } \\
\text { Bazo }\end{array}$ & Gastrointestin & $\begin{array}{c}\text { alCerebro } \\
\text { Hígado }\end{array}$ \\
\hline $\begin{array}{l}\text { Numero de } \\
\text { metástasis }\end{array}$ & & $1-4$ & $4-8$ & $>8$ \\
\hline $\begin{array}{l}\text { Bajo riesgo: 0-6 } \\
\text { puntos }\end{array}$ & & & & \\
\hline $\begin{array}{l}\text { Alto riesgo: } 7-13 \\
\text { puntos } \\
\text { Ultra alto riesgo: } \\
\text { más de } 13 \text { puntos }\end{array}$ & \multicolumn{4}{|c|}{$\begin{array}{l}\text { Bajo riesgo: tratamiento metotrexato } \\
\text { Alto riesgo: tratamiento poliquimioterapia }\end{array}$} \\
\hline
\end{tabular}

ciclos con otro ciclo de 6 semanas de refuerzo. La exploración ginecológica y las últimas pruebas de imagen realizadas (ecografía ginecológica y tomografía computarizada) no muestran evidencia de enfermedad a los 6 meses.

\section{Discusión}

El coriocarcinoma es un tumor gestacional perteneciente al grupo de enfermedades trofoblásticas 
gestacionales y con gran capacidad de invadir vasos, lo que facilita su diseminación y la aparición de metástasis regionales, pulmonares, cerebrales y en otros órganos. Las metástasis pulmonares son las más frecuentes $(80 \%)$, seguidas de las vaginales $(30 \%)$, las hepáticas y las cerebrales (10\%). En caso de metástasis hepáticas o cerebrales es necesario realizar una búsqueda exhaustiva de metástasis pulmonares. Desde el punto de vista etiológico se encuentra asociado con la edad, el grupo sanguíneo del sistema $\mathrm{ABO}$, abortos previos, uso de anticonceptivos orales y factores ambientales todavía no aclarados ${ }^{3}$. Para su diagnóstico y la valoración de la respuesta al tratamiento es muy importante determinar la $\beta$-hCG, porque unos valores elevados orientan la enfermedad y el seguimiento evolutivo de sus cifras ofrece una visión de la efectividad del tratamiento.

El tratamiento de primera línea del coriocarcinoma de bajo riesgo (Tablas 1-2) es la quimioterapia con metotrexato ${ }^{4}$, añadiendo etopósido, vincristina y actinomicina/ciclofosfamida en el caso del coriocarcinoma de alto riesgo. La quimioterapia debe continuarse hasta que se negativice la $\beta$-hCG, y luego se debe administrar un ciclo de consolidación. Durante todo el tratamiento hay que monitorizar la respuesta con determinaciones seriadas de la $\beta$-hCG. La histerectomía no se recomienda como tratamiento de primera línea. Sin embargo, puede ser considerada antes de la quimioterapia cuando no existen metástasis o en pacientes sin deseos de futuras gestaciones. La histerectomía como tratamiento coadyuvante a la quimioterapia puede plantearse en pacientes con enfermedad local, paridad cumplida o coriocarcinoma resistente a la quimioterapia La supervivencia es del $85-94 \%$ si no existe enfermedad metastásica, pero con metástasis la supervivencia disminuye según el órgano afectado ${ }^{5,6}$.

En nuestro caso, debido al deseo de futuras gestaciones y a la presencia de enfermedad metastásica, se optó por realizar cesárea y posterior quimioterapia.

\section{Conclusión}

El coriocarcinoma es un tumor del tejido placentario de baja incidencia y muy sensible a la quimioterapia. La clínica se debe tanto al propio tumor como a su producción hormonal. La $\beta$-hCG constituye el pilar fundamental para su diagnóstico y para la valoración de la respuesta al tratamiento. El tratamiento de primera línea consiste en la administración de quimioterapia, reservándose la histerectomía para casos seleccionados. Pese a ser un tumor muy agresivo, la tasa de supervivencia es alta, pero disminuye según la afectación de otros órganos.

\section{Financiamiento}

Los autores declaran que no existen fuentes de financiación públicas o privadas en la realización del presente artículo.

\section{Conflicto de intereses}

Los autores declaran que no existe ningún potencial conflicto de intereses relacionado con el artículo.

\section{Responsabilidades éticas}

Protección de personas y animales. Los autores declaran que para esta investigación no se han realizado experimentos en seres humanos ni en animales.

Confidencialidad de los datos. Los autores declaran que han seguido los protocolos de su centro de trabajo sobre la publicación de datos de pacientes.

Derecho a la privacidad y consentimiento informado. Los autores han obtenido el consentimiento informado de los pacientes y/o sujetos referidos en el artículo. Este documento obra en poder del autor de correspondencia.

\section{Bibliografía}

1. Seckl MJ, Seblre NJ, Berkowitz RS. Gestational trophoblastic disease. Lancet. 2010;376:717-29.

2. Sebire N, Seckl M. Gestational trophoblastic disease. En: Paul M, Lichtenberg ES, Borgatta L, Grimes DA, Stubblefield PG, Creinin MD, editores. Management of unintended and abnormal pregnancy. UK: BlackweII Publishing; 2009. p. 293- 301.

3. Ma Y, Xiang Y, Wan XR, Chen Y, Feng FZ, Lei CZ, et al. The prognostic analysis of 123 postpartum choriocarcinoma cases. Int J Gynecol Cancer. 2008;18:1097-101

4. Farley J, Heathcock B, Branch W, Larsen W, Homas D. Treatment of metastatic gestational choriocarcinoma with oral methotrexate in a combat environment. Obstet Gynecol. 2005;105:1250-4.

5. Altieri A, Silvia Franceschi JF, Smith J, Vecchia CL. Epidemiology and aetiology of gestational trophoblastic diseases. Lancet Oncol. 2003;4:670-8.

6. Seckl M, Seribe R, Fisher RA, Golfier F, Massuger L, Sessa C; ESMO Guidelines Working Group. Gestational trophoblastic disease: ESMO Clinical Practice Guidelines for diagnosis, treatment and follow-up. Ann Oncol. 2013;24 (Suppl 6):139-50. 